\title{
Is intraabdominal pressure a predictive factor for mortality and morbidity in abdominal trauma?
}

\author{
Daniel Ion***, Dan Nicolae Păduraru* **, Florentina Mușat*, Octavian Andronic* **, \\ Alexandra Bolocan * ** \\ *"Carol Davila" University of Medicine and Pharmacy, Bucharest, Romania \\ **University Emergency Hospital, Bucharest, Romania
}

Correspondence to: Dan Nicolae Păduraru, MD, PhD, University Emergency Hospital, Bucharest, 169 Splaiul Independentei Street, District 5, Bucharest, Romania, Mobile phone: +40744756 443, E-mail: dan.paduraru.nicolae@gmail.com

\begin{abstract}
The clinical signs and symptoms of an acute in increase intraabdominal pressure (IAP) are subtle, especially in the conditions of the polytraumatized patient. Thus, abdominal compartment syndrome (ACS) can brutally occur and can have a major impact on the body's main organs and systems. The purpose of our research was to identify the influence of intraabdominal pressure, intra-abdominal hypertension, and abdominal compartment syndrome, in the evolution of polytraumatized patients. Our study analyzed the patients admitted in the IIIrd Department of General Surgery of University Emergency Hospital in Bucharest between 1st of January 2010 and 31st of December 2018. The value of intraabdominal pressure, on admission, correlated with the risk of IAH/ACS in patients with abdominal trauma - being major causes of morbidity and mortality. IAP monitoring should become a mandatory part of the management plan for patients with abdominal trauma.

Keywords: abdominal trauma, intraabdominal pressure, intraabdominal hypertension, abdominal compartment syndrome
\end{abstract}

\section{Introduction}

The difficulty of therapeutic decisions in the management of patients with abdominal trauma or polytrauma with main abdominal component, in association with intraabdominal hypertension (IAH), is due to the fact that most of the times, the clinical signs and symptoms of an acute increase of intraabdominal pressure (IAP) are subtle, especially in the conditions of the polytraumatized patient. Thus, abdominal compartment syndrome (ACS) can brutally occur and can have a major impact on the body's main organs and systems.

On the other hand, the indication for laparotomy can be dictated either by the consequences of the traumatic lesions or by the pathophysiological consequences caused by the IAH syndrome. Thus, there is an increased risk of the occurrence of unnecessary/ nontherapeutic laparotomies.

Under these circumstances, we considered important to concentrate our efforts on improving the care of the patient with abdominal trauma in general, and, especially, in those who develop IAH syndrome. The purpose 
of our research was to identify the influence of intraabdominal pressure, intra-abdominal hypertension, and abdominal compartment syndrome, in the evolution of polytraumatized patients.

\section{Materials and methods}

Our study analyzed the patients admitted in the IIIrd Department of General Surgery of
University Emergency Hospital in Bucharest between 1st of January 2010 and 31st of December 2018, who met the inclusion criteria (Table 1). All the patients in the study were diagnosed at admission with abdominal trauma or polytrauma with main abdominal component and were managed, initially, using a conservative approach.

Table 1. Inclusion criteria specific for the patient with abdominal trauma or polytrauma with main abdominal component

\section{INCLUSION CRITERIA}

- Availability in the medical charts of all variables necessary for monitoring a patient with IAH/ ACS

- Minimum age of 18 years

- IAP measured at admission > $5 \mathrm{mmHg}$

- Abdominal injury was acquired through contusion

- Patient managed with a non-operative approach

- Patient with no contraindications for urinary catheter insertion

- Conscious, cooperative, hemodynamically stable or metastable patient

\section{Results}

The study involved a total of 121 patients who met the inclusion criteria. Depending on the presence or absence of IAH/ ACS in their evolution, we divided them into two groups:

1. Control group = Patients who did not develop IAH syndrome during hospitalization (86 cases).

2. IAH/ACS group = Patients who developed IAH syndrome during hospitalization (35 cases).

Regarding the therapeutic approach, in the control group, 72 patients $(83.72 \%)$ of the total of 86 cases were conservatively managed, while 14 patients (16.28\%) underwent surgery. In 9 cases the laparotomy was non-therapeutic/ unnecessary, while in the rest of 5 patients we identified lesions that needed surgical treatment.

Within the group of 35 patients who developed IAH/ ACS (28 - IAH, 7 - ACS), 16 patients were surgically managed (45.71\%). Of the 28 patients who developed IAH, 12 patients were surgically managed. Of the 7 patients who developed ACS, 4 patients underwent surgery.
Depending on the result of the laparotomy we were able to divide the surgical approach in therapeutic laparotomy - 8 cases, nontherapeutic/ unnecessary laparotomy - 2 cases, decompression laparotomy - 6 cases.

From the total of 121 patients 85 were males (70.24\%) and 36 were females (29.76\%). The average age of the patients in both the control group and the group of patients who developed IAH/ ACS was around 38 years old. Thus, we noticed the predominant involvement of the young population within these groups regardless of the occurrence or absence of IAH/ ACS during hospitalization.

Most abdominal trauma and polytrauma with main abdominal component were produced by road accidents and, in this context, 76 patients were in the position of the driver, the passenger or the pedestrian, of which 53 from the control group and 23 from the IAH/ ACS group. $2 \%$ of the cases were caused by motorcycle accidents. The following as frequency were precipitations while human aggression represented $13,22 \%$.

Following the analysis of the 121 patients 
based on the most important inclusion criterion (IAP > 5mmHg), we identified IAP values at admission varying between 5 and $13 \mathrm{mmHg}$. Moreover, there was a statistically significant correlation between the IAP value at the time of admission and the occurrence of IAH/ ACS in the evolution of patients with abdominal trauma or polytrauma with main abdominal component.

In our research, we identified 88 patients (representing $72.72 \%$ ) of the total number of 121 patients who had only abdominal trauma, while 33 patients (27.28\%) met the conditions of polytrauma.

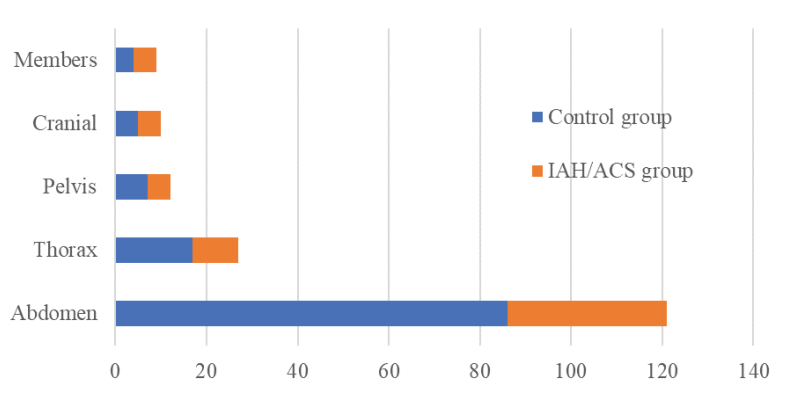

Fig. 1 Distribution of posttraumatic lesions in the study group

Considering the inclusion criteria in the study, all the patients experienced abdominal injury. The number of injuries identified in the patients enrolled in the study decreased in the following order: thorax, pelvis, central nervous system, and extremities (Fig. 1).

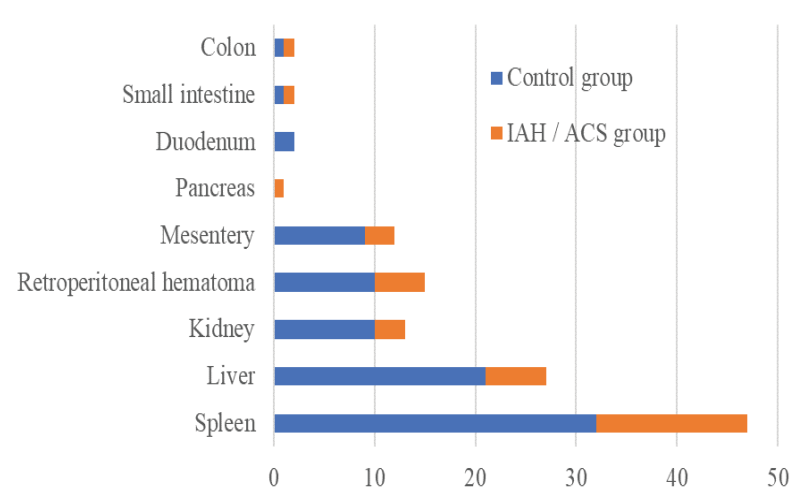

Fig. 2 Distribution of major abdominal lesions in the study group
In the majority of the cases, the most severely injured abdominal organ (the lesion was evaluated with the highest Abbreviated Injury Scale) was the spleen (38.84\%).

In order to facilitate the patient evaluation and to determine the best therapeutic approach of a patient with abdominal trauma or polytrauma with main abdominal component in the general surgery clinic, we considered appropriate to calculate an index that we named lesion score. We defined the lesion score as the number of injured sites in a case of polytrauma. In the case of the patient with abdominal trauma only, the lesion score was 1. For each site (thoracic, pelvic, craniocerebral, extremities) injured, we added 1 point at this index (Example: for a patient with abdominal, thoracic and pelvic lesions, the lesion score was 3) (Fig. 3).

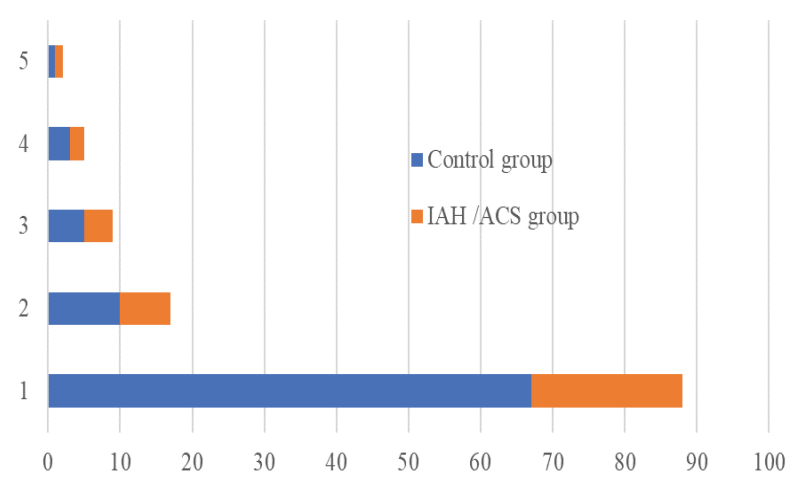

Fig. 3 Distribution of the lesion score in the study group

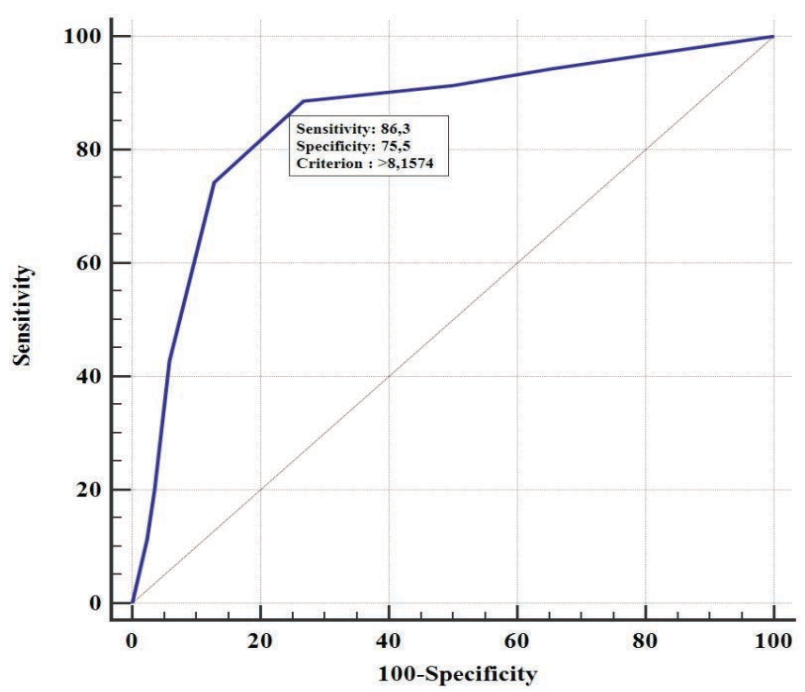


Under the area of the ROC curve

Standard deviation

Confidence interval (95\% probability)

z calculated

Threshold for statistical significance

(Area $=0.5$ )

Fig. 4 Correlation of intraabdominal pressure at admission with IAH syndrome during hospitalization

The value of IAP at the time of admission was a predictive factor for the occurrence of $\mathrm{IAH}$ in the evolution of a patient with

0,853

0,0406

0,778 to 0,911

8,715

$<0,0001$
Table 2. Statistical data regarding the total number of hospital days

\begin{tabular}{llllll}
\hline Hospital days & Average & Median & Standard deviation & Minimum & Maximum \\
TOTAL & 10,25 & 10 & 2,5894 & 5 & 14 \\
IAH & 9,64 & 10 & 2,4959 & 5 & 14 \\
ACS & 12,71 & 13 & 1,0301 & 11 & 14 \\
\hline
\end{tabular}

At the end of the study, 35 patients who developed $\mathrm{IAH}$ syndrome during admission summed up 359 hospital days with an average of 10.25 days. The average number of hospital abdominal trauma or polytrauma with main abdominal component (Fig. 4). During the assessment of the studied group, we identified and followed three severity markers in the evolution of patients with abdominal trauma/ polytrauma with main abdominal component who developed IAH syndrome:

- Total number of hospital days

- Number of days of care in the intensive care unit

- Mortality

Table 3. Statistical data regarding the number of intensive care unit days

\begin{tabular}{llllll}
\hline Intensive care unit days & Average & Median & $\begin{array}{l}\text { Standard } \\
\text { Deviation }\end{array}$ & Minimum & Maximum \\
TOTAL & $\mathbf{4 , 4 2}$ & 5 & $\mathbf{2 , 8 8 1 3}$ & $\mathbf{0}$ & 9 \\
IAH & $\mathbf{4 , 4 6}$ & 5 & $\mathbf{2 , 6 7 9 0}$ & 0 & 9 \\
ACS & $\mathbf{4 , 2 8}$ & 4 & 3,5742 & 0 & 9 \\
\hline
\end{tabular}

In the group of 35 patients with abdominal trauma or polytrauma with main abdominal component who developed IAH/ ACS, we identified 29 patients (82.85\%) who needed care in the intensive care unit. The total number of days spent at the intensive care unit was 155. The average number of days of care for patients who developed IAH was 4.46 while for the group of patients who developed ACS was 4.28 days (Table 3 ).

The mortality rate among patients who developed ACS was $28.57 \%$ (2 patients), while there were no deceased patients among patients with IAH.

By analyzing in a comparative manner the group of patients with abdominal trauma/ polytrauma with main abdominal component days for patients who developed IAH was 9.64, while for the patients with ACS was 12.71 days (Table 2).

who developed IAH/ ACS and the group of patients who did not develop IAH/ACS (Control Batch), we found that between the two groups there were significant differences in terms of the three severity evolution markers. The presence of $\mathrm{IAH} / \mathrm{ACS}$ in the evolution of the analyzed patients represented in our research a factor that strongly influenced mortality and morbidity (Fig. 5). 


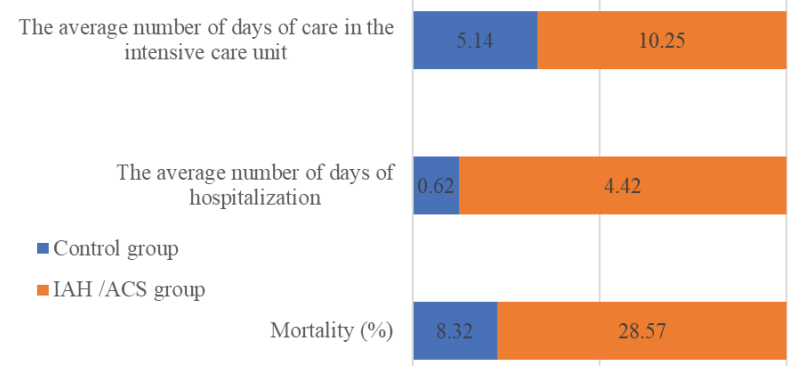

Fig. 5 Comparative analysis of control and IAH/ACS groups from the perspective of mortality and morbidity

\section{Discussion}

- Although most general surgeons have extensive knowledge of abdominal trauma management and have consistently made efforts to reduce the rate of unnecessary laparotomies, the therapeutic attitude for a patient with abdominal trauma is frequently directed towards exploratory laparotomy. Our research encourages this perspective in patients who develop IAH syndrome because the rate of unnecessary/ nontherapeutic laparotomies is extremely low in the group of these patients.

- Abdominal trauma is an entity that poses numerous problems regarding the therapeutic approach. In its severe form, often associated with IAH syndrome, it is a real challenge for the medical team that manages such a patient, because the causes are multiple, the pathophysiology is complex and incompletely deciphered, the optimal moment for setting up the surgical treatment is controversial, and nonoperative treatment is most often nonspecific and non-standardized. Under these circumstances, a rapid assessment of these types of patients with a scoring system containing only 6 parameters can quickly guide the clinician to the correct type of management.

- Non-operative management of IAH, both in the context of the patient with abdominal trauma and the patient with polytrauma with the main abdominal component, should be considered as replacing the morbidity associated with unnecessary laparotomies with other potential complications. One of the dreaded risks of this type of management is to misdiagnose a gastrointestinal tract lesion that may lead to a late set up of any therapeutic measures [1].

- Increased IAP is most often determined in the patient with abdominal trauma by tissue edema, which is a consequence of ischemia/ reperfusion phenomenon, sepsis, ileus, intra-/retroperitoneal hematoma, pneumoperitoneum and fluid resuscitation [2]. Treatment requires correction of the causes that led to an increase in IAP, such as intraabdominal/ retroperitoneal hemorrhages, post-traumatic ileus, or peritoneal collections. Under these circumstances, the decision to establish a certain type of management is extremely difficult. Thus, the rating system proposed by us can be very useful largely applied.

- In our research, comorbidities were rare $(14.04 \%$ of the patients). It is known that the outcome is worse in the case of traumas involving elderly people, especially due to preexisting co-morbidities [3]. Also, the literature reports data on the impact of comorbidities on morality, which we have not identified in our research. The highest impact on mortality is due to liver disease $(O R=5.1)$, renal disease $(O R=3.1)$, neoplasia $(O R=1.8)$ and chronic corticosteroid use (OR = 1.6) [4].

- In literature, a laparotomy type called unnecessary laparotomy is described. This includes laparotomy that does not identify any intraabdominal injury (negative laparotomy) and laparotomy that finds a minor lesion that does not argue for an additional surgical gesture (non-therapeutic laparotomy) [5]. The incidence of non-therapeutic laparotomies (NTLs) varies between 1.7 
and $38 \%$ depending on the experience and protocols of different trauma centers [6]. In our study, the nontherapeutic/ unnecessary laparotomy rate was $64.28 \%$ in the control group. Within the group of patients who developed IAH syndrome during hospitalization, the proportion of nontherapeutic laparotomies was $12.5 \%$.

\section{Conclusions}

1. In order to identify cases that associate $\mathrm{IAH}$ syndrome during hospitalization, in the management of patients with abdominal trauma/ polytrauma with main abdominal component it is necessary to monitor IAP starting from the hospital admission.

2. Considering that a large number of patients with abdominal trauma develop high levels of IAP, we believe that the management of those patients should also take into account the effects that IAH may have on the evolution of these cases.

3. The value of intraabdominal pressure, on admission, correlates with the risk of $\mathrm{IAH} /$ ACS in patients with abdominal trauma - being major causes of morbidity and mortality.

4. IAP monitoring should become a mandatory part of the management plan for patients with abdominal trauma.

\section{Acknowledgements \\ Funding \\ Not applicable.}

\section{Authors' contributions}

All authors have contributed equally in writing, reading, and approving the final manuscript.

\section{Consent for publication}

Not applicable.

\section{Competing interests}

Not applicable.

\section{Conflict of Interest statements}

Authors state no conflict of interest.
Informed Consent and Human and Animal Rights statements

Informed consent has been obtained from all individuals included in this study.

\section{Authorization for the use of human subjects}

Ethical approval: The research related to human use complies with all the relevant national regulations, institutional policies, is in accordance with the tenets of the Helsinki Declaration, and has been approved by the authors' institutional review board or equivalent commitee.

\section{References}

1. De Waele JJ, Ejike JC, Leppäniemi A, De Keulenaer BL, De Laet I, Kirkpatrick AW, Roberts DJ, Kimball E, Ivatury $\mathrm{R}$, Malbrain ML. Intra-abdominal hypertension and abdominal compartment syndrome in pancreatitis, paediatrics, and trauma. Anaesthesiol Intensive Ther. 2015; 47(3):219-27. doi: 10.5603/AIT.a2015.0027.

2. Strang SG, Van Imhoff DL, Van Lieshout EM, D'Amours SK, Van Waes OJ. Identifying patients at risk for highgrade intra-abdominal hypertension following trauma laparotomy. Injury. 2015 May; 46(5):843-8. doi: 10.1016/j.injury.2014.12.020.

3. Ertel W, Oberholzer A, Platz A, Stocker R, Trentz O. Incidence and clinical pattern of the abdominal compartment syndrome after "damage-control" laparotomy in 311 patients with severe abdominal and/or pelvic trauma. Crit Care Med. 2000; 28:1747-1753.

4. Dubose JJ, Scalea TM, Holcomb JB, Shrestha B, Okoye $\mathrm{O}$, Inaba $\mathrm{K}$ et al. Open abdominal management after damage-control laparotomy for trauma: a prospective observational American Association for the Surgery of Trauma multicenter study. J Trauma Acute Care Surg. 2013; 74:113-120(discussion 1120-1122).

5. Chiara O, Cimbanassi S, Biffl W, Leppaniemi A, Henry S, Scalea TM, Catena F, Ansaloni L, Chieregato A, de Blasio E, Gambale G, Gordini G, Nardi G, Paldalino P, Gossetti F, Dionigi P, Noschese G, Tugnoli G, Ribaldi S, Sgardello S, Magnone S, Rausei S, Mariani A, Mengoli F, di Saverio S, Castriconi M, Coccolini F, Negreanu J, Razzi S, Coniglio C, Morelli F, Buonanno M, Lippi M, Trotta L, Volpi A, Fattori L, Zago M, de Rai P, Sammartano F, Manfredi R, Cingolani E. International consensus conference on open abdomen in trauma. J Trauma Acute Care Surg. 2016 Jan; 80(1):173-83. doi: 10.1097/TA.0000000000000882. Review.

6. Mahmood I, Mahmood S, Parchani A, Kumar S, El-Menyar A, Zarour A, Al-Thani H, Latifi R. Intra-abdominal hypertension in the current era of modern traumaresuscitation. ANZ J Surg. 2014 Mar; 84(3):166-71. doi: 10.1111/ans.12169. 